\title{
Causal Reasoning Mining Approach to Analog Circuit Verification
}

\author{
Fanshu Jiao*, Alex Doboli \\ Department of Electrical and Computer Engineering \\ State University of New York at Stony Brook, Stony Brook, NY 11794-2350
}

\begin{abstract}
Functional errors in analog portion of mixed signal circuits become more severe and improvements in verification methods are increasingly important. Current verification methods fall into two categories, simulation-based verification and formal verification [1], focusing on verifying analog circuit function/performance. This paper proposes a novel approach verifying analog circuit design using causal reasoning. Causal reasoning is the inductive reasoning process to create a new design. The flow begins with mining the causal reasoning steps (design plan) that produced the circuit, including starting ideas, design step sequence, and their justifications [2]. Then, topological features corresponding to the starting ideas and design step sequence are verified individually by replacing the related devices with ideal behavior model. Performance is evaluated through Cadence Spectre simulation. Comparison with new circuit performance reveals incorrect functional issues and/or performance potentials for improvement. They are negative causes of certain starting ideas or design steps, which might have been omitted during the design process. The paper discusses three operational amplifier designs realized in $0.2-\mu \mathrm{m}$ CMOS technology to illustrate the verification approach.
\end{abstract}

Keywords: analog verification, causal reasoning, CMOS Op-amp

\section{Introduction}

Modern analog circuits in advanced CMOS technologies are more complex and with more demanding performance constraints. In traditional system on chips (SoCs), analog and mixed-signal circuitry take up $20 \%$ of the area, but this percentage is expected to steadily increase to half of the chip area in SoCs designed for advanced nodes [3]. An increasing number of functional errors and unmet performance requirements occur in the analog portion of large mixed-signal integrated chips [4]. Design refinement and redesigns are more severe in order to meet time-to-market requirements, hence driving the need for consistent analog verification methodologies and tools. Analog verification requires performing functional verification on analog, mixed-signal and RF integrated circuits and SOCs, which still depends largely on designer's experience and expertise. For digital systems, there are various reliable verification methods, i.e. formal verification of

\footnotetext{
${ }^{*}$ Corresponding author

Email addresses: fanshu.jiao@stonybrook.edu (Fanshu Jiao), alex.doboli@stonybrook.edu (Alex Doboli)
}

RTL. These methods do not directly apply to analog circuits, not only because analog signals process continuously in value and time, but also the complexity of analog performance specifications. While existing methods, simulation-based verification and formal verification, aim at covering and verifying the entire performance space, they arguably offer less diagnostics insight, i.e. the reasons that cause the performance errors.

This paper proposes a new verification approach for analog circuits based on design causal reasoning. While designing a new circuit, the design flow complies with certain intuitive reasoning procedure. By understanding and mining design reasoning, we are able to utilize the information to verify functional errors and potentials for improvement. Design causal reasoning finds the most likely circuit design plan that produced a circuit, i.e. what ideas served as starting points and how the design steps were carried out from the starting ideas. The verification method checks the correctness of the starting ideas and design steps that form a design plan. Incorrect functional issues and performance potentials for improvement are identified and linked to either starting ideas and/or design steps. The result of verification offers diagnostics insight, like the starting idea and/or 
design step implementation that should be modified to correct functional errors.

Design causal reasoning can be reused or extended to tackle new problems, also help understand how design ideas evolve within a design community [2]. Uncovering and understanding causal reasoning has been an important research topic in biological, neuroscience, data analysis fields. [5] studies the causality between two simultaneously recorded biological signals, and investigates whether one signal is causing the other. [6] studies causal relations to model gene interactions. [7] proposes an extension to Granger causality [8], to measure how different brain regions may causally influence on each other. Identifying analog circuit design steps is similar to understanding system or process causal reasoning. Though causal reasoning study has not been performed in analog design automation, it is helpful for understanding the design process and design verification purpose.

The paper is organized as follows: Section 2 discusses the related work. Section 3 briefly presents the algorithm to mine circuit causal reasoning, and then describes the verification flow. Section 4 presents two experiments on verifying Op-amps to show how design issues and improvement potentials are identified. Conclusions in Section 5 end the paper.

\section{Related Work}

Recent research on analog circuit verification can be distinguished into two categories: simulation-based methods and formal verification methods [1].

Simulation-based methods verify a system or a circuit design at various levels of abstraction of the analog or mixed-signal (AMS) domain. Transient simulation, a main tool in AMS verification, generally requires long simulation time and large computing resources. Simulation time can be reduced by using behavioral models of the circuits $[9,10,11]$. Existing behavioral modeling methods include black box modeling $[12,13]$, white box or structural modeling $[10,11]$, base-band modeling, and event-driven modeling [14].

For RF circuits, there are specific, enhanced simulation methods, i.e. periodic steady-state analysis and harmonic balance. However, these methods are not applicable to AMS circuits which are not periodic [15]. Base-band modeling isolates the modulated data from the carrier frequency. The main issue with base-band modeling is using different pin definitions while switching between different abstraction levels. Base-band modeling tools like SPW [16], SpectreRF [17] and simulator AMS designer [18] support the verification of a RF subsystem at different levels of abstractions. [19] uses enhanced base-band behavioral models for an industrial-level multiband, low IF GSM receiver. Eventdriven modeling approach isolates the high-frequency signal path from the low-frequency bias and control by using different simulation domains. The event-driven simulation approach in [20] verifies RF front-ends using a new double precision data type (called wreal) in Verilog-AMS. It separates high frequency signal paths and saves time by enabling fast analog verification using the digital simulation domain. Simulation-based verification is widely used, but limited by the fact that it is hard to identify input patterns that guarantee the covering of all errors that might occur in the whole design space.

Formal verification methods, though have not been widely applied to AMS and RF design verification, can mathematically verify a circuit's operation and/or performance space. Existing formal verification methods include model checking, reachability analysis, equivalence checking, and runtime verification. Performance properties are described in the steady-state space. A first attempt to formulate analog simulation as a Boolean satisfiability (SAT) problem is proposed in [21]. [22] extends the SAT-based modeling method to parameter variations. Conventional temporal logic model for AMS circuit verification is discussed in [23]. It applies finite state machines to represent continuous state space of transient response. [24] proposes two symbolic model checking algorithms, a binary decision diagram-based model checker and a symbolic modular-based model checker. Both model checkers utilize a Boolean symbolic model derived from the labeled hybrid petri net model. It includes Boolean signals to represent digital circuitry and continuous variables to model voltages and currents of analog circuits. Besides various other model checking approaches, [25] introduces dynamic stability verification by zonotope-based reachability analysis. Steinhorst et al [26] present an algorithm for behavioral equivalence checking of two circuit implementations for dynamic state space. Model-checking and reachability analysis validate the design over a range of parameters and input possibilities, therefore suffers from expensive computation [27]. [28] presents a runtime verification algorithm using a time-augmented rapidlyexploring random tree for incremental runtime monitoring.

In conclusion, current simulation-based and formal verification methods mainly focus on verifying circuit performance, but arguably offer less diagnostics insight, i.e. the reasons that cause the errors. 


\section{Analog Circuit Verification Flow}

Each analog circuit design follows certain design reasoning procedure, which can be extracted by using the procedure in [2] applied to the circuit design literature. Mining design reasoning identifies possible design features that served as starting ideas in devising new circuits as well as the design steps and their justifications in creating the complete designs. The proposed causal reasoning-based verification flow begins with mining design causal reasoning from the circuit design literature. Circuit topological features corresponding to starting ideas, design steps are verified, then the diagnostics information is obtained. Figure 1 shows the verification flow.

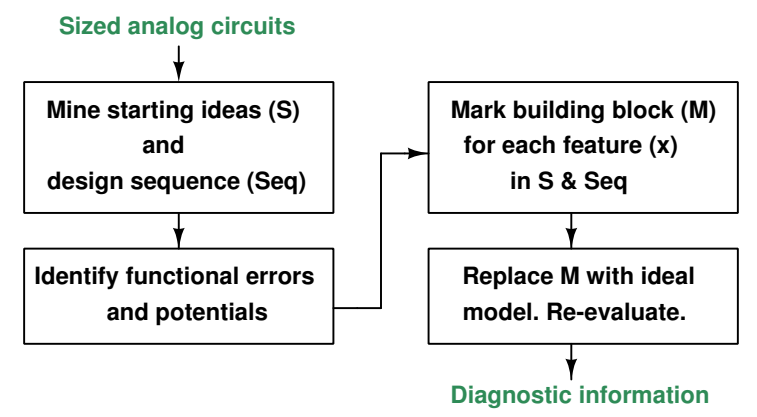

Figure 1: Causal reasoning-based verification flow

\subsection{Mining causal reasoning}

As explained in [2], the main elements describing design causal reasoning include starting ideas, design steps, and justifications. The specifications are the objectives for optimization. This subsection summarizes the main ideas discussed in [2].

Starting ideas are the most likely design features and constraints that served as starting points in devising a circuit design [2]. They introduce the topological features or abstract design features that originate the design sequence to create a given circuit. Note that the very initial ideas (e.g. ideas from previous design experiences) that have jump-started a new design process might be different from starting ideas. The insight gained during an iterative, reasoning-based design process transform and evolve the very initial ideas into the starting ideas. By analyzing the design literature, we classified starting ideas into four categories based on their origins and defined four sets. Private set (set $\Gamma$ ) includes ideas that have been used by the same group in their previous circuit designs and appeared in the current circuit. Collective set (set $\Lambda$ ) includes ideas that occur in other groups' designs and which are present in the analyzed circuit too. Such ideas are inferred from the literature citation list. New design insight (set $\Phi$ ) includes new insight ideas created by the designer, which cannot be found in any related publication. Common set (set $\Theta$ ) incorporates ideas are well-known, traditional design knowledge in textbooks. All the topological features (building blocks) of a circuit are extracted as set $\Sigma$. A design sequence is a succession of design steps, justified by either improving certain performance trade-offs and bottlenecks, or relaxing solution constraints.

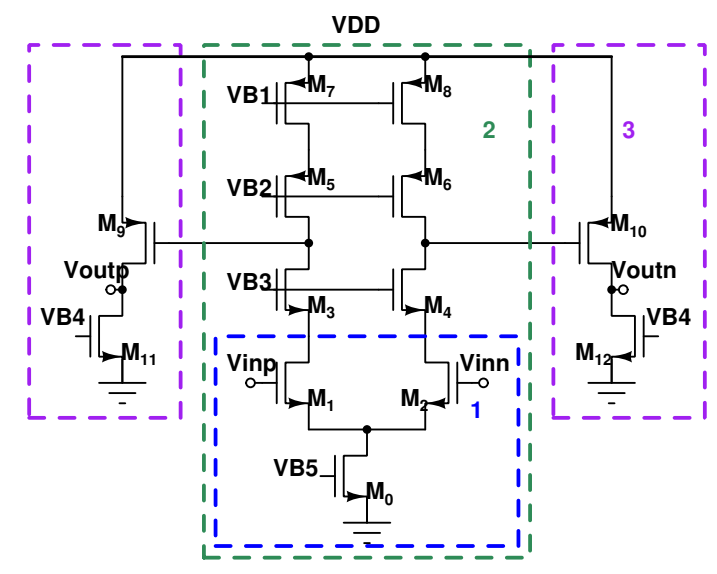

Figure 2: Two stage Op-amp schematic

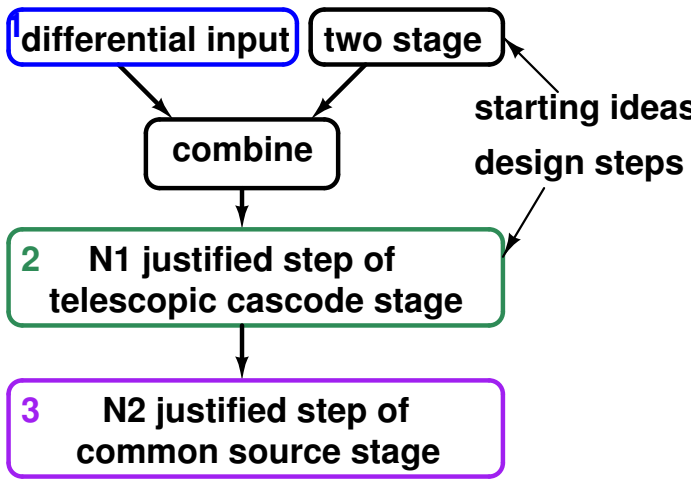

Figure 3: Two-stage Op-amp causal reasoning

Initially, starting ideas (set $S$ ) are the union of set $\Gamma$ and set $\Lambda$, ideas used in previous designs by the same designer and by other designers that are cited in the current solution. The starting idea can be topological feature (correspond to a physical implementation) or abstract feature (can be realized by various topological features). E.g., "Two stage" is an abstract feature, as two stage corresponds to various physical implementations. The starting ideas are not necessarily equal to the 
final topological features (building blocks) in the circuit. If the starting idea is topological, the designer might use it directly in the new design (set $S$ and $\Sigma$ overlap). If the starting idea is abstract, the designer might derive his own way of implementation based on the abstract idea (set $S$ and $\Sigma$ do not overlap). If the difference $\Sigma-S$ is not empty then for each such feature $x$ in $\Sigma-S, x$ is identified as part of the design sequence of the circuit, if $x$ is derived by a starting idea in $S(S \rightarrow x)$. A new design step $N$ is created to reflect the changes in the design introduced by adding feature $x$, its implementation and the modification in performance and design constraints. $N$ is added to design sequence. Design steps related to biasing are added too. The algorithm ends if no more design steps $N$ are added to design sequence. The remaining unjustified features in $\Sigma$, if any, are added to set $S$, as they are either ideas presenting designer's new insight (set $\Psi$ ) or common design knowledge (set $\Theta$ ).

Example: Figure 2 shows the schematic of a two stage Op-amp circuit [29]. The complete topological features (building blocks) of the circuit ( $\operatorname{set} \Sigma)=$ \{differential input, telescopic cascode stage, common source stage\}, labeled 1, 2, 3 in Figure 2. The initial starting ideas (set $S$ ) = \{an abstract feature two-stage , which has been used in previous design as indicated by the author [29] (set $\Gamma$ ). Beginning with the initial starting idea in set $S$, the uncovered features $=\Sigma-S$. In this case, set $\Sigma$ and $S$ don't overlap, as the designer used the concept of two-stage but decided his own physical implementations. Two-stage is analyzed further to derive design sequence. \{Telescopic cascode stage, common source stage\}, labeled as 2 and 3, the two-stage implementations, are mined as design sequence. Therefore, design step N1 implements the first gain stage as telescopic cascode stage, justified by its advantages in high gain. Design step N2 implements the second gain stage as common source stage, as it offers high output voltage swing. The remaining features, differential input, labeled 1, is common design knowledge. Therefore, it is added to set $S$ as the starting ideas. Set $S$ includes all starting ideas of the circuit and the identified design sequence includes all features in set $\Sigma-S$, hence it produces the final design. Figure 3 illustrates the mined starting ideas and the design step sequence.

\subsection{Circuit verification using causal reasoning}

The causal reasoning-based verification method is shown in Algorithm 1. It links circuit functional errors or performance potentials for improvement with the corresponding starting ideas/design steps in the causal reasoning. Verification results are a set of functional errors, potentials for improvement, and the features in design that produced them (the diagnostics information).

For a sized circuit, the verification flow first compares performance simulation results with specifications. In the experiment, we sized the circuit using Cadence advanced analysis optimization tool [30]. The specifications in Table 2 are the objectives for optimization. Performances are evaluated through Spectre simulator. Starting from the mined causal reasoning information, the topological features in the starting ideas and design sequence are individually verified. Features are verified from starting ideas to design sequence as starting ideas are considered more important than the design sequence. For each topological feature $x$, the corresponding related devices are identified as a block, as labeled in Figure 2. Each block performs certain function and might have drawback insight. Replacing the block with an ideal functional model removes the drawbacks and sometimes functional errors in the circuit.

A two terminal Verilog-AMS behavioral amplifier model [31] is chosen as the ideal functional model. It has only two parameters and simplifies the verification process. Two model parameters are gain and input offset. Gain is set as the same with the replaced block to keep the new circuit having the same gain as the original. Input offset is set to zero.

For each performance $p$, if $p$ is marked as functional error but $p$ is solved in the new circuit $F$ using the ideal functional model, then feature $x$ (belonging to starting ideas or design sequence) is the reason (or part of reason) for $p$ not meeting the specification. Otherwise, if performance $p$ is relaxed in $F$, it indicates that we can look for better candidate of feature $x$ to improve $p$.

Example: The circuit in Figure 2 was sized in $0.2 \mu \mathrm{m}$ CMOS technology. All transistor L dimensions are chosen 3 times the minimum size. Table 1 lists the optimized sizing solution.

The overall performance set $=\{$ static power, DC gain, bandwidth, gain-bandwidth product (GBP), slew rate, rmsNoise, total harmonic distortion (THD), output voltage swing\}. Two figures of merit $\mathrm{FOM}_{S}$ and $\mathrm{FOM}_{L}$ were used to characterize small-signal and large-signal performances [32]. All circuits have load capacitor $\mathrm{C}_{\text {load }}=10 \mathrm{pF}$. Therefore, we considered to verify the performance set $\left\{\mathrm{FOM}_{S}\right.$, Phase Margin, $\mathrm{FOM}_{L}$, rmsNoise, THD, output voltage swing\}. The simulation results in Table 2 show that phase margin, $\mathrm{FOM}_{L}$ and noise don't meet the specification, which is shown in bold and 


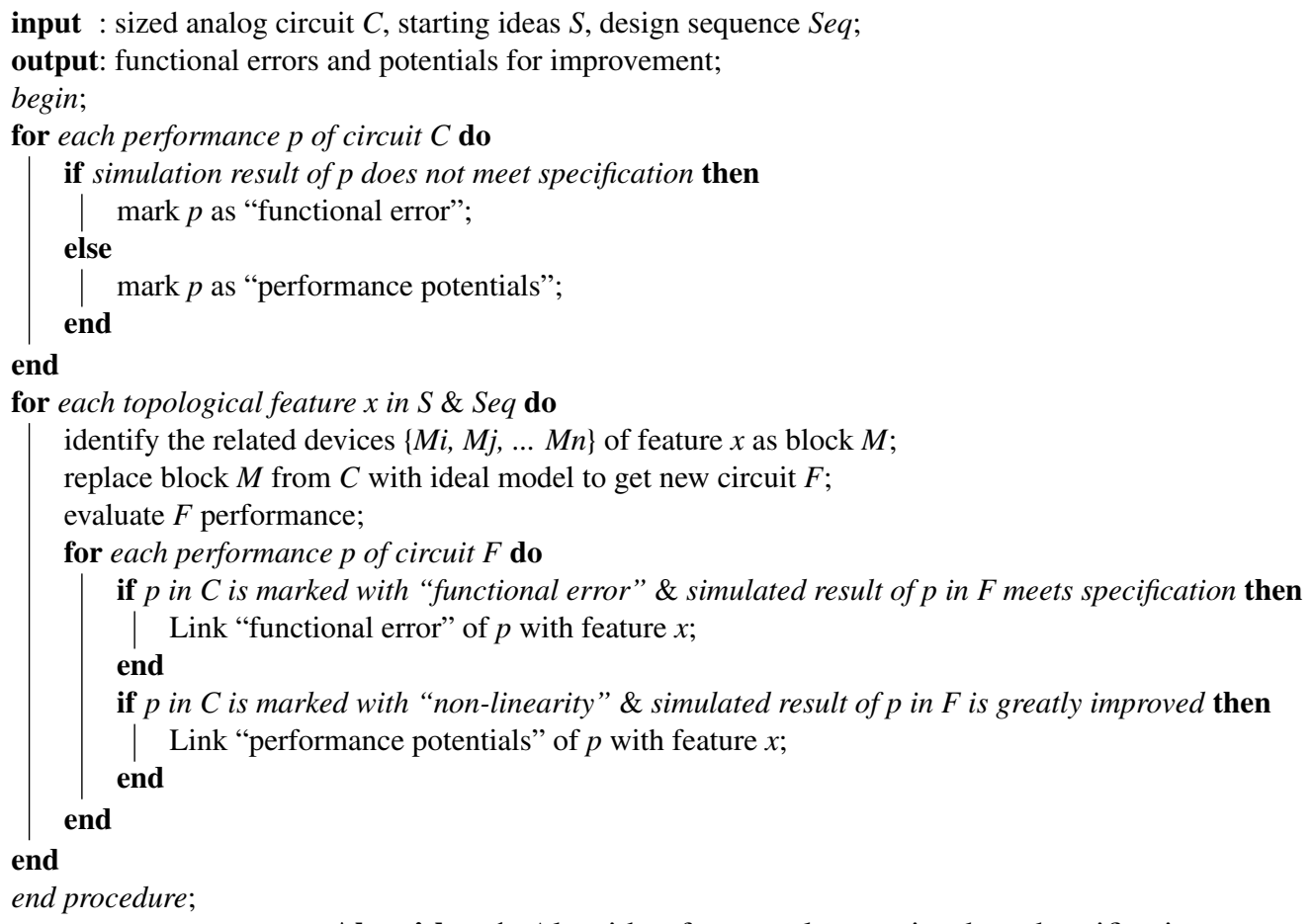

Algorithm 1: Algorithm for causal reasoning-based verification

Table 1: Transistor sizing for the Op-amp circuit in Figure 2

\begin{tabular}{|c|c|c|c|c|c|c|c|}
\hline devices & M0 & M1/M2 & M3/M4 & M5/M6 & M7/M8 & M9/M10 & M11/M12 \\
\hline optimized sizing $[\mu / \mu]$ & $15 / 0.6$ & $69.75 / 0.6$ & $90 / 0.6$ & $14.6 / 0.6$ & $26.8 / 0.6$ & $1.1 / 0.6$ & $0.3 / 0.6$ \\
\hline
\end{tabular}

marked as "functional error".

$$
F O M_{S}=\frac{G B P * C_{L}}{\text { power }}, F O M_{L}=\frac{S R * C_{L}}{\text { power }}
$$

The verification flow for each topological feature in the starting idea and design sequence in Figure 2, labeled as 1,2, and 3, is as follows.

Feature 1. Differential input is identified as devices $\{M 0, M 1, M 2\}$. Replacing $\{M 0, M 1, M 2\}$ with the ideal amplifier model (shown as a triangle) generates a new circuit $F 1$ in Figure 4(a). The ideal model has the same gain with the block being replaced. The input offset is set to $0 \mathrm{~V}$. Column $F 1$ in Table 2 summarizes the newly evaluated performances of circuit $F 1$. With respect to performance set $\left\{\mathrm{FOM}_{S}\right.$, phase margin, $\mathrm{FOM}_{L}$, rmsNoise, THD, and output voltage swing\}, phase margin is slightly improved, but doesn't meet the specification. Feature differential input introduces one nondominant pole. Noise in the new circuit is greatly reduced. Thus we conclude that feature differential input introduces most of the noise. If improvement on noise is needed, we will focus on input stage and look for better candidate. Topological feature differential input, as one of the starting ideas, introduces one non-dominant pole, thus is not the reason for having insufficient phase margin.

Feature 2. The telescopic cascode stage is implemented as the first gain stage in the amplifier. The new circuit $F 2$ in Figure 4(b) is generated by replacing the related devices $\{M O \sim M 8\}$. In circuit $F 2$, phase margin meets the constraint, so one dominant pole introduced by the first stage is eliminated. $\mathrm{FOM}_{S}$ and rmsNoise improved significantly as the new circuit replaced the entire first stage, including devices in differential input.

Feature 3. The second gain stage of the amplifier is implemented by a common source stage. Replacing the related devices $\{M 9 \sim M 12\}$ results in the new topology in Figure 4(c). Comparing performance results, phase margin >90 means gain will not drop to zero within the considered frequency range. $\mathrm{FOM}_{L}$ is greatly improved. We can conclude that the second stage introduces one dominant pole. Also, the second stage greatly constrains the small signal and large signal performance.

In summary, the two-stage telescopic Op-amp suffers from insufficient phase margin, low large signal perfor- 

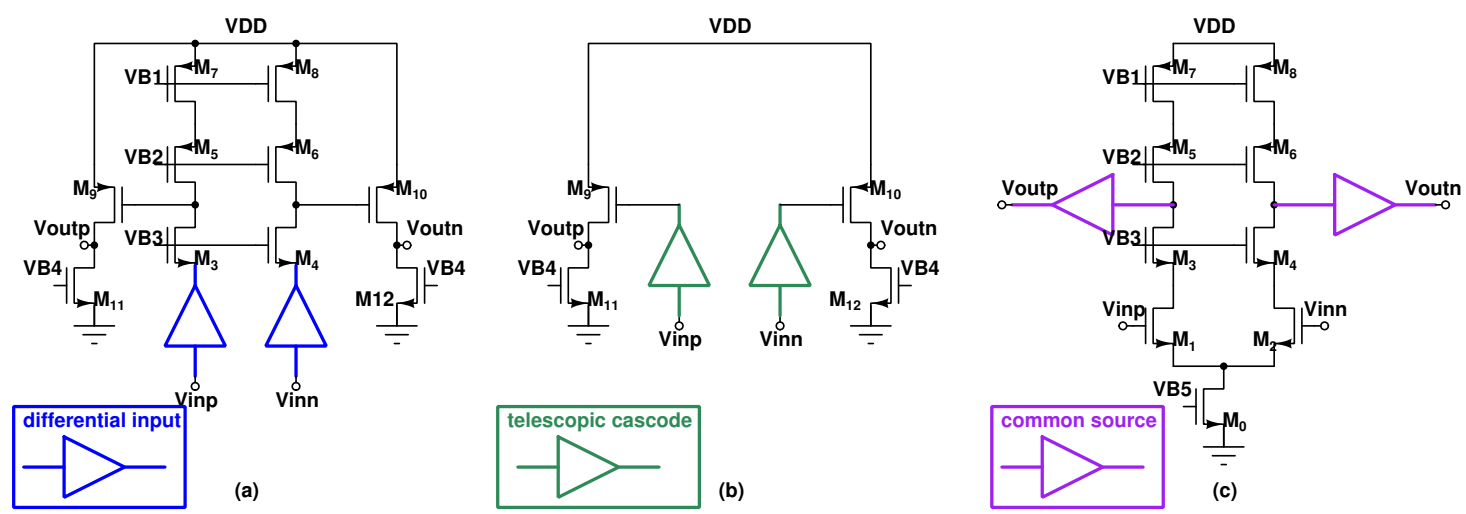

Figure 4: Replace circuit features in Figure 2 with models

Table 2: Performance comparison of circuits in Figure 2, 4

\begin{tabular}{|c|c|c|c|c|c|}
\hline & & & & \\
\hline Performance & Specs & Optimized results & F1 & F2 & F3 \\
\hline Static power $[\mu \mathrm{w}]$ & minimize & 405.072 & 405.096 & 8.212 & 396.84 \\
\hline DC gain[dB] & maximize & 89.79 & 89.98 & 89.93 & 89.39 \\
\hline Bandwidth [kHz] & maximize & 6.245 & 6.125 & 6.153 & $2.921 \mathrm{e} 3$ \\
\hline GBP [MHz] & maximize & 193.2 & 193.7 & 193.6 & $86.3 \mathrm{e} 3$ \\
\hline $\mathbf{F O M}_{S}\left[\frac{M H z^{*} p F}{m w}\right]$ & $>100$ & 4769.5 & 4781.6 & 235752.6 & 2174680 \\
\hline Phase Margin $\left[{ }^{\circ}\right]$ & {$[60,90]$} & 3.4 & 13.32 & 81.68 & $>90$ \\
\hline Pos Slew Rate $[\mathrm{V} / \mu \mathrm{s}]$ & $>1$ & 0.46 & 0.47 & 0.38 & $17.36 \mathrm{e} 3$ \\
\hline $\mathbf{F O M}_{L}\left[\frac{V / \mu * p F}{m w}\right]$ & $>100$ & 11.32 & 11.51 & 467.85 & 437455.9 \\
\hline rmsNoise [1-1MHz band] & $<10$ & 27.82 & 4.75 & 0.63 & 32.76 \\
\hline THD [\%] & $<10$ & 1.35 & 1.11 & 1.09 & 1.75 \\
\hline Output voltage swing [V] & $>1.2$ & 1.6 & 1.6 & 1.6 & 1 \\
\hline
\end{tabular}

mance and large noise. By analyzing the two stages, we conclude there are two dominant poles in the circuit which cause the phase drop. One is after the telescopic cascode stage and one is at the output load. With respect to the three topological features in the starting ideas and design sequence, the two-stages implementation in the design sequence needs to be modified. Additional feedforward stage or frequency compensation is required to ensure stability. The starting idea on using differential input contributes most on noise, and the idea of using the output stage constrains most large signal performance (slew rate).

\section{Experiments}

Experiments illustrate the proposed verification flow for two more advanced OTA/Op-amps.

\subsection{Highly linear OTA verification}

The circuit in Figure 5 is a single differential highly linear OTA with degeneration resistance [33]. Set $\Sigma$ of the circuit $=\{$ differential input, resistance degeneration,

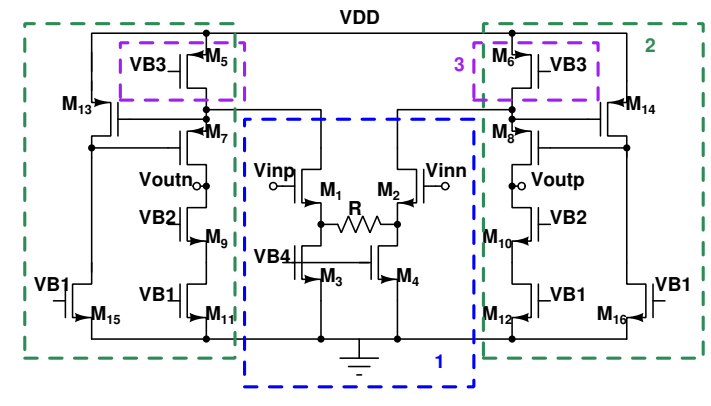

Figure 5: Two stage OTA schematic

regulated cascode, and current source biasing $\}$. Set $S$ $=\{$ source degenerated differential input $\}$, which is referred from previous work (set $\Gamma$ ). It is labeled as 1 in Figure 5. It offers high linearity by using higher source degeneration factors. The uncovered features $(\Sigma-S)$ are labeled as 2 and 3. The uncovered features, \{regulated cascode, current source biasing\}, if justified, are added to the design sequence of the circuit. Source degenerated differential input achieves higher linearity than regular differential input but limits the gain, thus regu- 


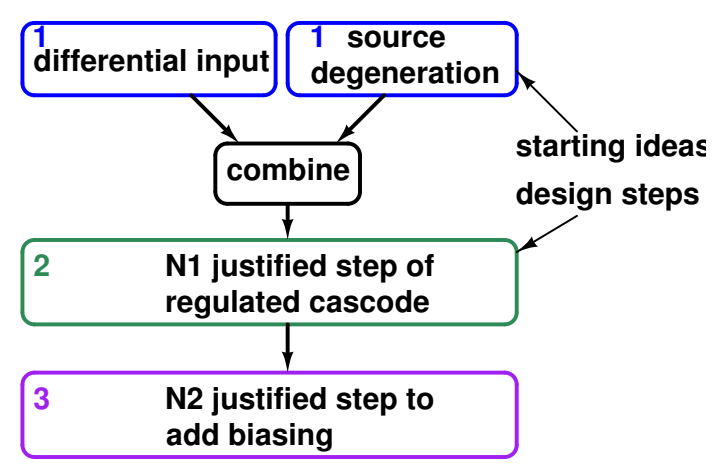

Figure 6: Two stage OTA casual reasoning

lated cascode is used at the output. Design step Nl adds regulated cascode, justified by the starting idea to boost gain. Current source biasing is a required design step for the circuit's correct operation. Figure 6 illustrates the mined starting ideas and the design sequence. From the performance comparison in Table 3, output voltage swing does not meet specification, and are marked as functional errors. Phase margin, rmsNoise, and THD are labeled performance potentials.

Feature 1. The topological structures in the starting idea, differential input and source degeneration, are identified as one building block, $\{M 1 \sim M 4\}$, and resistor $R$. The circuit with ideal model is shown in Figure 7(a). $\mathrm{FOM}_{S}$ is greatly improved, but the output voltage swing is still low. The input stage implementation is part of the reasoning for $\mathrm{FOM}_{S}$ improvement. The other performances do not have a significant improvement, thus the input stage does not introduce limitations on other performances.

Feature 2. The design step uses regulated cascode as the output stage. The new circuit in Figure 7(b) solves the functional error. The design step corresponding to the regulated cascode stage is the mainly reason for the small- and large signal performance improvement. It also dominates the output voltage swing. Same as with the input stage, it does not introduce constrains on other performances.

Feature 3. Step N2 is a required design step. Biasing transistors $M 5$ and $M 6$ are modeled by the ideal current sources in Figure 7(c). According to Table 3, it does not have a major effect on performance. The simulation errors come from the transistor non-idealities working as current sources, which is not for verification purposes.

For low voltage application, using the regulated cascode implementation as the output stage should be avoided. Both gain stages cause not sufficient $\mathrm{FOM}_{S}$ and $\mathrm{FOM}_{L}$, whereas the output stage dominates more on $\mathrm{FOM}_{L}$.

\subsection{High-speed, high-swing Op-amp}

Another circuit example in Figure 8 is a highspeed, high-swing Op-amp used in low-voltage applications [34]. From the circuit topology, we extracted five topological features (building blocks) $=\{$ differential in put single ended output, gain-boosted amplifier, folded cascode, low voltage current mirror, and current source biasing\} (set $\Sigma$ ). These five topological features are labeled as $1 \sim 5$ in Figure 8. According to the design description in [34], the reasoning process begins with ideas used previously, which is gain boosted (feedback) amplifier (set $\Gamma$ ). For the remaining features in $\Sigma-S$, folded cascode is justified by the feedback amplifier. The folded cascode with feedback path is one strategy to enhance gain with additional power consumption. The step of adding current source biasing is a required design step. Differential input single ended output is a common input-output structure (set $\Theta$ ). Meanwhile, low voltage current mirror is one way to connect differential input and single output stage, which is a justified design step. The causal reasoning in Figure 9 combines the starting ideas of differential input, single ended output and gain-boosted amplifier (set $\Gamma$ ), labeled as 1 and 2 . Design step $N 1$ implements the input stage using folded cascode. Design step $N 2$ adds a low-voltage current mirror justified by the single-ended output. Design step N3 adds the current source biasing for the circuit's correct operation. They are labeled as 3, 4, and 5 in Figure 8.

As shown in Table 4, rmsNoise and output voltage swing are unmet performances. Figure 10 presents five new circuits generated by replacing each feature in set starting ideas and design sequence. From column Fl, small signal and large signal performance are improved greatly, whereas noise does not change.

Design steps of using folded cascode and low voltage current mirror, and the starting idea of using gainboosted amplifier are the main reasons for the unsatisfied noise requirement. Steps of folded cascode and low-voltage current mirror determines output voltage swing, which is easy to conclude as they form the output stage. Differential input introduces performance drawbacks on small- and large signal performance.

The presented verification examples identify functional errors, potential for improvement and their reasons, e.g., incorrect starting ideas or design steps (diagnostics) for a optimally sized circuit. The insight obtained from verification examples can be used as a reference for further design. Hence, in contrast to many of the existing methods, the proposed technique also finds the origin of functional errors in a circuit. 

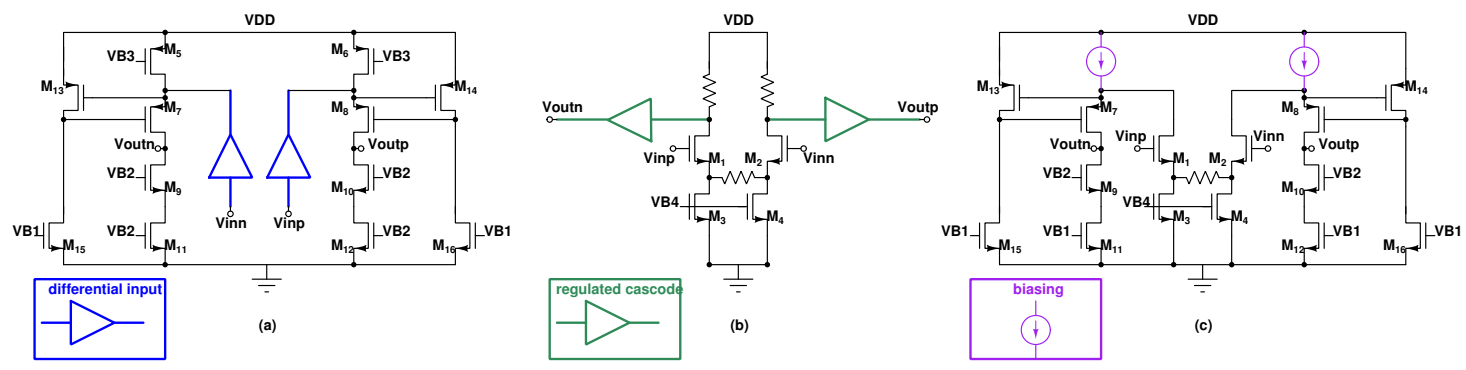

Figure 7: Replace circuit features in Figure 5 with models

Table 3: Performance comparison of circuits in Figure 5, 7

\begin{tabular}{|c|c|c|c|c|c|}
\hline Performance & Specs & Optimized results & F1 & F2 & F3 \\
\hline Static power $[\mu \mathrm{w}]$ & minimize & $1.017 \mathrm{e} 3$ & $1.017 \mathrm{e} 3$ & 456 & $1.25 \mathrm{e} 3$ \\
\hline DC gain $[\mathrm{dB}]$ & maximize & 38.16 & 38.23 & 30.17 & 33.04 \\
\hline Bandwidth [kHz] & maximize & 382.2 & $14.89 \mathrm{e} 3$ & $468.9 \mathrm{e} 6$ & 69.78 \\
\hline GBP $[\mathrm{MHz}]$ & maximize & 30.99 & $1.217 \mathrm{e} 3$ & $15.15 \mathrm{e} 3$ & 3.14 \\
\hline $\mathbf{F O M}_{S}\left[\frac{M H z^{*} p F}{m w}\right]$ & $>100$ & 304.65 & 11961.86 & 332236.84 & 25.11 \\
\hline Phase Margin $\left[{ }^{\circ}\right]$ & {$[60,90]$} & 88.8 & 67.9 & $>90$ & 91.11 \\
\hline Pos Slew Rate $[\mathrm{V} / \mu \mathrm{s}]$ & $>1$ & 13.88 & 12.67 & $8.293 \mathrm{e} 3$ & 3.908 \\
\hline $\mathbf{F O M}_{L}\left[\frac{V / \mu * p F}{m w}\right]$ & $>100$ & 136.45 & 124.533 & 181864.04 & 31.25 \\
\hline rmsNoise [1-1MHz band] & $<10$ & 0.3394 & 0.02711 & 0.09976 & 1.836 \\
\hline THD $[\%]$ & $<10$ & 1.011 & 1.048 & 1.071 & 0.9783 \\
\hline Output voltage swing [V] & $>1.2$ & 1.2 & 1.2 & 2 & 1.2 \\
\hline
\end{tabular}
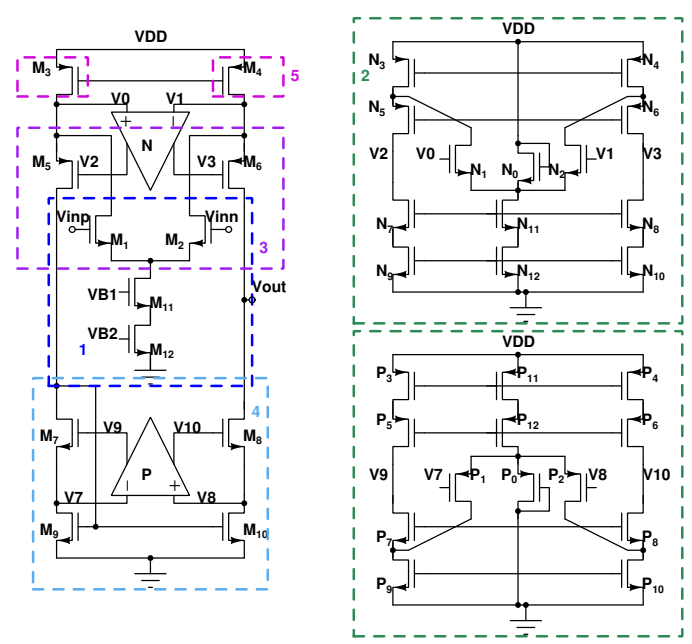

Figure 8: High-speed and high-swing op-amp

\section{Conclusions}

This paper presents a new causal reasoning-based approach on verification of analog circuits to identify functional errors and performance potentials for improvement and their causes (diagnostics). The verification flow begins with mining the causal reasoning steps (design plan) that produced the circuit, including start-

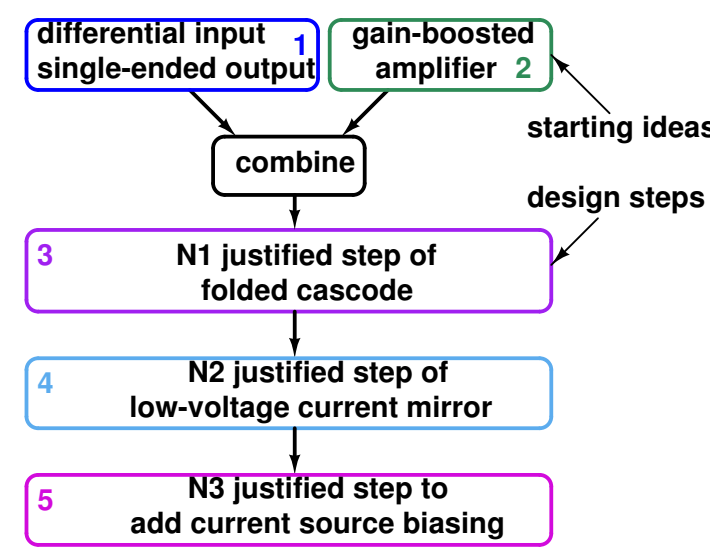

Figure 9: High-speed and high-swing op-amp causal reasoning

ing ideas, design step sequence, and their justifications. Topological features corresponding to the starting ideas and design step sequences are verified individually by replacing the related devices (transistors, capacitors, resistors, etc) with ideal behavior model. Comparing simulation results with the new generated circuits reveals incorrect functional issues and/or performance potential for improvement. They are negative causes of certain starting ideas or design steps, which might have been omitted during the design process. Three exper- 

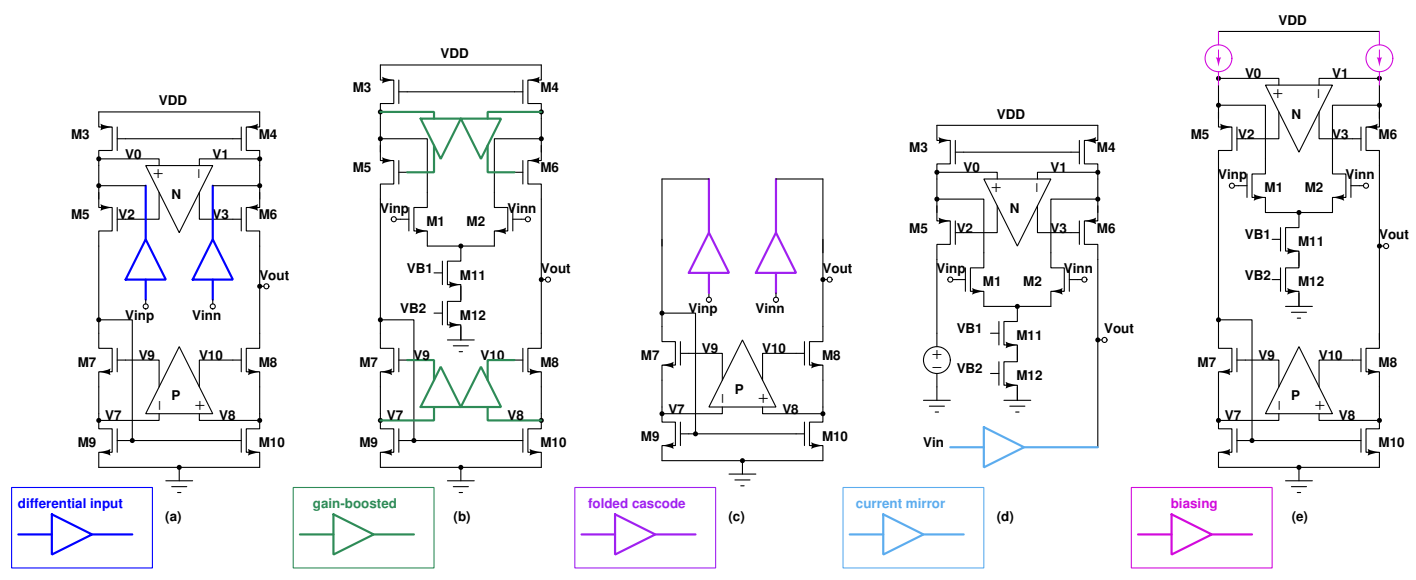

Figure 10: Replace circuit features in Figure 8 with models

Table 4: Performance comparison of circuits in Figure 8, 10

\begin{tabular}{|c|c|c|c|c|c|c|c|}
\hline Performance & Specs & Optimized results & F1 & F2 & F3 & F4 & F5 \\
\hline Static power $[\mu \mathrm{w}]$ & minimize & 994.44 & 994.44 & 738.8 & 0 & 738.8 & 752 \\
\hline DC gain $[\mathrm{dB}]$ & maximize & 95.3 & 95.06 & 15.09 & 95.3 & 79.6 & 97.7 \\
\hline Bandwidth [kHz] & maximize & 0.508 & 34.23 & 1023 & $+\infty$ & $+\infty$ & 0.3872 \\
\hline GBP $[\mathrm{MHz}]$ & maximize & 29.64 & 1943 & 5.831 & $+\infty$ & $+\infty$ & 29.77 \\
\hline $\mathbf{F O M}_{S}\left[\frac{M H z * p F}{m w}\right]$ & $>100$ & 298.06 & 19538.63 & 78.93 & $+\infty$ & $+\infty$ & 395.88 \\
\hline Phase Margin $\left[{ }^{\circ}\right]$ & {$[60,90]$} & 86.01 & 75 & 106.4 & $>90$ & $>90$ & 86.9 \\
\hline Pos Slew Rate $[\mathrm{V} / \mu \mathrm{s}]$ & $>1$ & 17.13 & 1517 & 0.0885 & $23.28 \mathrm{e} 9$ & 1198 & 17.3 \\
\hline $\mathbf{F O M}_{L}\left[\frac{V / \mu * p F}{m w}\right]$ & $>100$ & 172.26 & 15254.82 & 1.65 & $+\infty$ & 16215.48 & 230.05 \\
\hline rmsNoise [1-1MHz band] & $<10$ & 240.1 & 199.3 & 0.076 & $\mathbf{0}$ & $\mathbf{0}$ & 273.6 \\
\hline THD $[\%]$ & $<10$ & 0.99 & 1.527 & 61.28 & 0.3 & 0.226 & 0.99 \\
\hline Output voltage swing [V] & $>1.2$ & 1 & 1 & 2 & 1 & 2 & 1 \\
\hline
\end{tabular}

iments on Op-amp designs show that functional errors and performance potentials are identified as either incorrect starting design ideas or erroneous design steps in the design plan. Automating the verification process includes extracting circuit building blocks, mining causal reasoning information [2], and evaluating new circuits with replaced ideal model. Extracting building blocks and mining causal reasoning have been discussed. Future work will focus on automating the performance evaluation on idealized models. Future work will also consider other design specifications, e.g., sensitivity analysis, second-order effects, and verification on other types of analog circuits.

\section{Acknowledgment}

This material is based upon work supported by the National Science Foundation under Grant BCS 1247971. Any opinions, findings, and conclusions or recommendations expressed in this material are those of the author(s) and do not necessarily reflect the views of the National Science Foundation.

\section{References}

[1] E. Barke, D. Grabowski, H. Graeb, L. Hedrich, S. Heinen, R. Popp, S. , Y. Wang, Formal approaches to analog circuit verification, in: Proc. DATE, EDAA, pp. 724-729.

[2] F. Jiao, S. Montano, C. Ferent, A. Doboli, S. Doboli, Analog circuit design knowledge mining: Discovering topological similarities and uncovering design reasoning strategies, IEEE Trans. on CADICS (2015).

[3] E. Naviasky, et al., Mixed-signal design challenges and requirements, Cadence white paper (2009).

[4] K. Kundert, H. Chang, Verification of complex analog integrated circuits, in: Proc. CICC, IEEE, pp. 177-184.

[5] M. El-Gohary, J. McNames, T. Ellis, B. Goldstein, Time delay and causality in biological systems using whitened crosscorrelation analysis, in: Engineering in Medicine and Biology Society, 2006. EMBS'06. 28th Annual International Conference of the IEEE, IEEE, pp. 6169-6172.

[6] O. Nikolova, S. Aluru, Parallel discovery of direct causal relations and markov boundaries with applications to gene networks, in: Parallel Processing (ICPP), 2011 International Conference on, IEEE, pp. 512-521.

[7] S. Hu, G. Dai, G. A. Worrell, Q. Dai, H. Liang, Causality analysis of neural connectivity: critical examination of existing methods and advances of new methods, Neural Networks, IEEE Transactions on 22 (2011) 829-844.

[8] C. Chen, A. Maybhate, D. Israel, N. V. Thakor, X. Jia, Assessing thalamocortical functional connectivity with granger causality, 
Neural Systems and Rehabilitation Engineering, IEEE Transactions on 21 (2013) 725-733.

[9] Y. Wei, A. Doboli, Systematic development of analog circuit structural macromodels through behavioral model decoupling, in: Proceedings of the 42nd annual Design Automation Conference, ACM, pp. 57-62.

[10] Y. Wei, A. Doboli, Systematic development of nonlinear analog circuit macromodels through successive operator composition and nonlinear model decoupling, in: Proceedings of the 43rd annual Design Automation Conference, ACM, pp. 1023-1028.

[11] Y. Wei, A. Doboli, Structural macromodeling of analog circuits through model decoupling and transformation, Computer-Aided Design of Integrated Circuits and Systems, IEEE Transactions on 27 (2008) 712-725.

[12] F. De Bernardinis, M. I. Jordan, A. SangiovanniVincentelli, Support vector machines for analog circuit performance representation, in: Design Automation Conference, 2003. Proceedings, IEEE, pp. 964-969.

[13] S. Doboli, G. Gothoskar, A. Doboli, Piecewise-linear modeling of analog circuits using trained feed-forward neural networks and adaptive clustering of hidden neurons, in: Neural Networks, 2003. Proceedings of the International Joint Conference on, volume 2, IEEE, pp. 1126-1131.

[14] Y. Wang, S. Joeres, R. Wunderlich, S. Heinen, Modeling approaches for functional verification of rf-socs: limits and future requirements, Computer-Aided Design of Integrated Circuits and Systems, IEEE Transactions on 28 (2009) 769-773.

[15] K. Kundert, Challenges in rf simulation, in: Radio Frequency integrated Circuits (RFIC) Symposium, 2005. Digest of Papers. 2005 IEEE, IEEE, pp. 105-108.

[16] R. Frevert, J. Haase, R. Jancke, U. Knochel, P. Schwarz, R. Kakerow, M. Darianian, Modeling and Simulation for RF System Design, Springer Science \& Business Media, 2006.

[17] Virtuoso spectre circuit simulator rf analysis user guide (2007).

[18] Cadence ams simulator user guide (2001).

[19] S. Joeres, S. Heinen, Functional verification of radio frequency socs using mixed-mode and mixed-domain simulations, in: Behavioral Modeling and Simulation Workshop, Proceedings of the 2006 IEEE International, IEEE, pp. 144-149.

[20] S. Joeres, H.-W. Groh, S. Heinen, Event driven analog modeling of rf frontends, in: Behavioral Modeling and Simulation Workshop, 2007. BMAS 2007. IEEE International, IEEE, pp. 46-51.

[21] S. K. Tiwary, A. Gupta, J. R. Phillips, C. Pinello, R. Zlatanovici, First steps towards sat-based formal analog verification, in: Computer-Aided Design-Digest of Technical Papers, 2009. ICCAD 2009. IEEE/ACM International Conference on, IEEE, pp. $1-8$.

[22] M. Miller, F. Brewer, Formal verification of analog circuit parameters across variation utilizing sat, in: Proceedings of the Conference on Design, Automation and Test in Europe, EDA Consortium, pp. 1442-1447.

[23] T. R. Dastidar, P. Chakrabarti, A verification system for transient response of analog circuits, ACM Transactions on Design Automation of Electronic Systems (TODAES) 12 (2007) 31.

[24] D. C. Walter, Verification of analog and mixed-signal circuits using symbolic methods, Ph.D. thesis, Citeseer, 2007.

[25] Y. Song, H. Yu, S. M. P. DinakarRao, Reachability-based robustness verification and optimization of sram dynamic stability under process variations, Computer-Aided Design of Integrated Circuits and Systems, IEEE Transactions on 33 (2014) 585-598.

[26] S. Steinhorst, L. Hedrich, Equivalence checking of nonlinear analog circuits for hierarchical ams system verification, in: Proc. VLSI-SoC, IEEE, pp. 135-140.

[27] M. H. Zaki, S. Tahar, G. Bois, Formal verification of analog and mixed signal designs: A survey, Microelectronics Journal 39 (2008) 1395-1404.

[28] S. N. Ahmadyan, J. A. Kumar, S. Vasudevan, Runtime verification of nonlinear analog circuits using incremental timeaugmented rrt algorithm, in: Proceedings of the Conference on Design, Automation and Test in Europe, EDA Consortium, pp. 21-26.

[29] B. Razavi, Design of analog CMOS integrated circuits, Boston, MA: McGraw-Hill, 2000.

[30] Cadence advanced analysis tools user guide (2002) 162-207.

[31] Cadence verilog-ams language reference (2005) 293.

[32] H. Lee, P. K. Mok, Active-feedback frequency-compensation technique for low-power multistage amplifiers, IEEE JSSC 38 (2003) 511-520.

[33] A. Lewinski, J. Silva-Martinez, Ota linearity enhancement technique for high frequency applications with im3 below-65 db, IEEE Trans. on CAS II: Express Briefs 51 (2004) 542-548.

[34] M. M. Ahmadi, A new modeling and optimization of gainboosted cascode amplifier for high-speed and low-voltage applications, IEEE Trans. on CAS II: Express Briefs 53 (2006) 169-173. 